\title{
The association of red and processed meat, and dietary fibre with colorectal cancer in UK Biobank
}

\author{
K.E. Bradbury and T.J. Key \\ Cancer Epidemiology Unit, Nuffield Department of Population Health, Oxford University, Oxford OX3 7LF
}

Marked international variation in both dietary patterns and colorectal cancer incidence rates ${ }^{(1)}$, coupled with the observation of changing colorectal cancer rates in migrant populations that have moved from one country to another ${ }^{(2)}$, suggests dietary and lifestyle factors play a role in the aetiology of colorectal cancer. Although some progress has been made in identifying dietary risk factors for colorectal cancer in existing large cohort studies, there is remaining uncertainty around the role of specific dietary factors, particularly meat ${ }^{(3)}$. UK Biobank provides the opportunity for detailed and comprehensive study of dietary intakes and colorectal cancer risk and will provide us with updated information in a UK population ${ }^{(4)}$.

The objective of the current study was to examine the associations between red meat, processed meat, and fibre with colorectal cancer in UK Biobank. UK Biobank is a prospective cohort of 500,000 men and women aged 40-69 years who were recruited from the UK in 2006-2010 and have been followed since. Participants reported their frequency of consumption of meat and other food groups on a touchscreen questionnaire at recruitment and a sub-sample of participants completed at least one 24-hour recall ${ }^{(5)}$ An estimate of fibre intake was derived from the touchscreen questions and calibrated using the 24-hour recall. Cases of colorectal cancer were identified through linkage with the National Health Service Central Registers. Cox regression models were used to estimate adjusted relative risks by the reported frequency of consumption of meat and fibre for colorectal cancer, stratified by sex, age, region, and deprivation, and adjusted for education, height, waist circumference, smoking, alcohol, and physical activity.

During a median of 2 years of follow-up, 1175 cases of colorectal cancer occurred. More than half of the study population were consuming more than the UK government recommendation of $70 \mathrm{~g}$ or less of red and processed meat per day. Reported consumption of meat was associated with an increased risk of colorectal cancer. The relative risk (RR) for reported consumption of red and processed meat 7 or more times per week vs once a week or less $=1.52,95 \% \mathrm{CI}$ : 1.13 to $2.06, p_{\text {trend }}=0.041$; the RR for reported consumption of red meat 4 or more times a week vs less than once a week $=1.44,95 \% \mathrm{CI}: 1.10$ to $1.89, p_{\text {trend }}=0.052$; the RR for reported consumption of processed meat 2 or more times per week vs never $=1 \cdot 36,95 \%$ CI 1.04 to $1 \cdot 77, p_{\text {trend }}=0 \cdot 026$. Estimated consumption of fibre was not significantly associated with colorectal cancer incidence (RR for highest fifth vs lowest fifth $=0.88,95 \%$ CI: 0.73 to $1 \cdot 06, p_{\text {trend }}=0 \cdot 23$ ). In these preliminary analyses from UK Biobank, consumption of red and processed meat was associated with an increased risk of colorectal cancer.

1. Armstrong B \& Doll R (1975) Int J Cancer 15, 617-631.

2. Maskarinec G \& Noh JJ (2004) Ethn Dis 14, 431-439.

3. Scientific Advisory Committee on Nutrition (2010) Iron and Health. London: TSO

4. Collins R (2012) Lancet 379, 1173-1174.

5. Liu B, Young H, Crowe FL, et al. (2011) Public Health Nutr 14, 1998-2005. 